\title{
Widely tunable resonant cavity enhanced detectors built around photonic crystals
}

\author{
Burak Temelkuran, Ekmel Ozbay
}




\title{
Widely Tunable Resonant Cavity Enhanced Detectors Built Around Photonic Crystals
}

\author{
Burak Temelkuran, and Ekmel Ozbay \\ Department of Physics, Bilkent University, Ankara, Turkey 06533
}

\begin{abstract}
We report a resonant cavity enhanced (RCE) detector built around a three-dimensional photonic band gap crystal. We have demonstrated the resonant cavity enhanced (RCE) effect by placing microwave detectors in defect structures built around dielectric and metallic based photonic crystals. We measured a power enhancement factor of 3450 for planar cavity structures built around dielectric based photonic crystals. The tuning bandwidth of the RCE detector extends from 10.5 to $12.8 \mathrm{GHz}$. We also demonstrated the RCE effect in cavities built around metallic structures. The power enhancement for the EM wave within these defect structures were measured to be around 190. These measurements show that detectors embedded inside photonic crystals can be used as frequency selective RCE detectors with increased sensitivity and efficiency when compared to conventional detectors.
\end{abstract}

Keywords: Photonic crystals, resonant cavity enhancement, resonators, detectors, resonant detectors

\section{INTRODUCTION}

Photonic crystals are periodic dielectric structures with energy band diagrams exhibiting a forbidden energy region for electromagnetic (EM) waves, similar to electronic band gaps that appear in semiconductor crystals. ${ }^{1-9}$ In the past few years, there were numerous attempts to fabricate photonic crystals at optical frequencies. ${ }^{10-12}$ However due to technological difficulties related to fabrication of sub-micron structures, photonic crystals with a full band-gap have been accomplished at only microwave, millimeter-wave, and far infrared frequencies. As an example, the layer-by-layer photonic crystal that was proposed by Ho et al. ${ }^{13}$ was successfully fabricated (with a full photonic band gap) at frequencies ranging from $15 \mathrm{GHz}$ to $30 \mathrm{THz}{ }^{14-16}$ Defects or cavities around the same geometry can also be built by means of adding or removing rods from the so called layer-by-layer photonic crystals. ${ }^{17}$ The electrical fields in such cavities are usually enhanced ${ }^{18}$ and by placing active devices in such cavities, one can make the device benefit from the wavelength selectivity and the large enhancement of the resonant EM field within the cavity. This effect has already been used in optoelectronics to achieve novel devices such as resonant cavity enhanced (RCE) photodetectors and light emitting diodes. ${ }^{19}$ In this paper, we demonstrate the RCE effect by placing microwave detectors within the localized modes of photonic crystal defect structures.

\section{STRUCTURE AND EXPERIMENT}

In our experiments, we used a layer-by-layer dielectric photonic crystal designed to have three-dimensional band gap with a midgap frequency around $12 \mathrm{GHz}$. The layer-by-layer photonic band gap crystal we have designed and fabricated consists of simple one-dimensional dielectric rods as the basic building blocks. ${ }^{13}$ The structure is assembled by stacking together layers of dielectric rods with each layer consisting of parallel rods with a center to center separation of $a$. The rods are rotated by $90^{\circ}$ in each successive layer. Starting at any reference layer, the rods of every second neighboring layer are parallel to the reference layer but shifted by a distance of $0.5 a$ perpendicular to the rod axes. This results in a stacking sequence that repeats every four layers. This lattice has face centered tetragonal (fct) lattice symmetry with a basis of two rods. The photonic band gap is not sensitive to the cross sectional shape of the rods. This structure has a photonic band gap when both the filling ratio and the dielectric contrast meet certain requirements. The layer-by-layer structure was constructed by using square-shaped alumina rods $(0.32 \mathrm{~cm} \times 0.32 \mathrm{~cm} \times 15.25 \mathrm{~cm})$. The photonic crystal has a center to center separation of $1.12 \mathrm{~cm}$, corresponding to a dielectric filling ratio of $\sim 0.29$. We used the output port of a microwave network analyzer and a standard gain horn to obtain EM waves. 


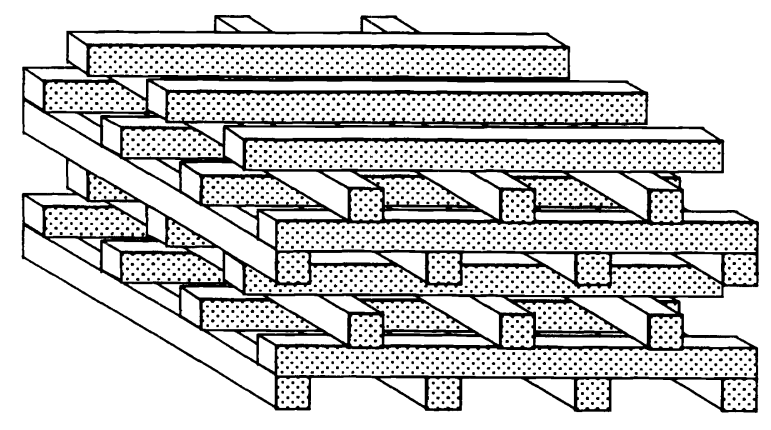

Figure 1. Schematics of face center tetragonal based photonic crystals

Defect structures built around the crystal were tested by putting them in the beam-path of the EM waves propagating along the stacking direction. A square law microwave detector was placed inside the defect volume of the photonic crystal, along with a monopole antenna. The monopole antenna was kept parallel to the polarization vector $\boldsymbol{e}$ of the incident EM wave in all measurements. The DC voltage on the microwave detector was used to measure the power of the EM field within the cavity. We also measured the enhanced field by feeding the output of the monopole antenna into the input port of the network analyzer. The monopole antenna was constructed by removing the shield around one end of a microwave coaxial cable. The exposed center conductor which also acted as the receiver, was $2 \mathrm{~mm}$ long. The calibrated enhancement measurements were performed in the following manner. We first measured the enhanced EM field by the probe inside the cavity. While keeping the position of the probe fixed, we removed the crystal and repeated the same measurement. This single pass absorption data of the probe was then used for calibration of the first measurement.

We first investigated a planar defect structure which was built around a 16 layer photonic crystal. The planar defect was obtained by separating the $8^{\text {th }}$ and $9^{\text {th }}$ layers of the structure. ${ }^{20}$ This resulted in a planar air-gap between two photonic mirrors, each formed of an 8-layer crystal. Figure 2(a) shows the enhancement characteristics of a planar defect structure with a separation width of $8.5 \mathrm{~mm}$. The measurement was done by the network analyzer and the frequency was chosen to cover the photonic band gap of our crystal. We observed a power enhancement factor of 1600 at a defect frequency of 11.68 GHz. The Q-factor (quality factor), defined as the center frequency divided by the full width at half-maximum, was measured to be 900 . We then measured the enhancement characteristics of the same defect structure (Figure 2(b)), with a microwave detector, inserted inside the same cavity. An enhancement factor of 450 along with a Q-factor of 1100, were observed at the same defect frequency.
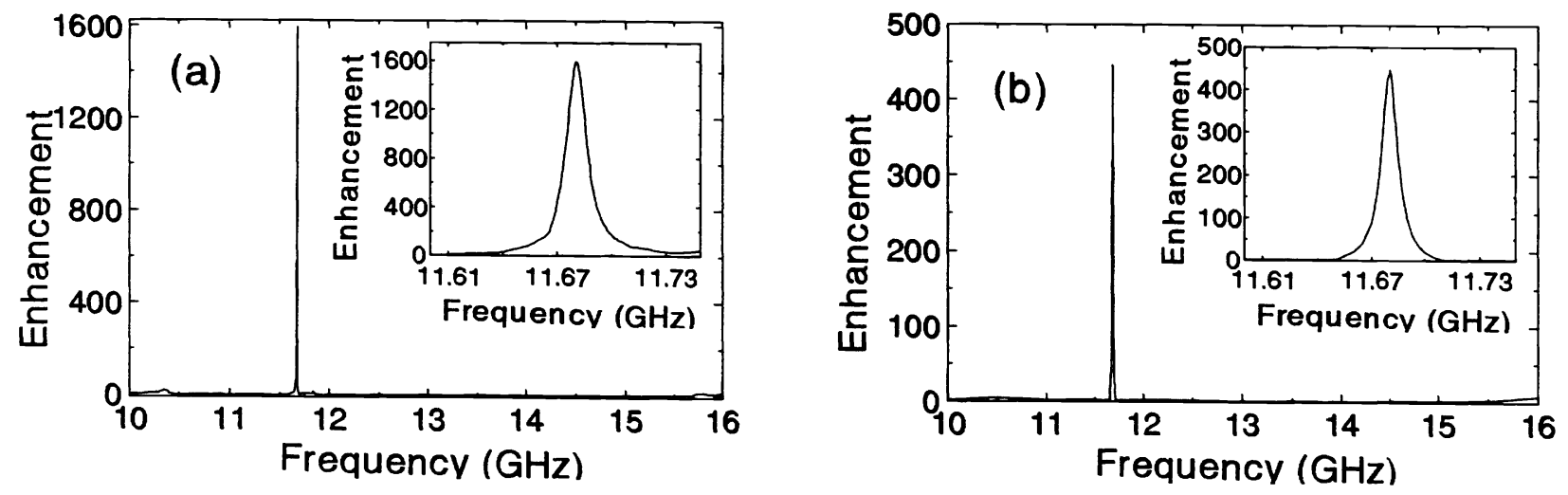

Figure 2. Experimental enhancement factors obtained for a planar defect structure: (a) using the network analyzer, (b) using the microwave detector. 


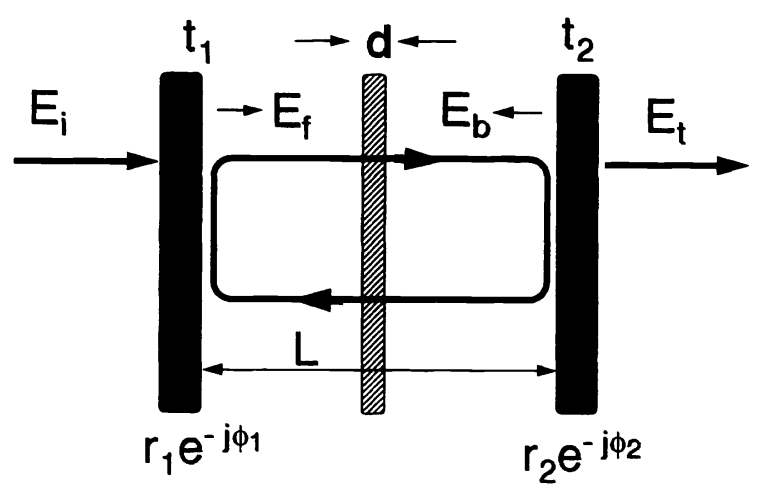

Figure 3. Schematics of the Fabry Perot cavity model. The shaded absorption region was used to simulate the detector placed in the cavity.

\section{THEORY}

The discrepancy between two measured enhancement factors can be explained by modeling our structure as a Fabry-Perot cavity $^{22}$ (Figure 3). The probe we used in our experiments was simulated by an absorption region of thickness d, with a relative absorption coefficient $\alpha$. The electric field component for the forward traveling wave $E_{f}$ inside the cavity can be related to the incident field $\mathrm{E}_{\mathrm{i}}$ as:

$$
E_{f}=\frac{t_{1}}{1-r_{1} r_{2} e^{-\alpha d} e^{-j\left(2 \beta L+\phi_{1}+\phi_{2}\right)}} E_{i}
$$

where $\mathbf{r}_{1} \mathbf{e}^{-\mathrm{j} \phi_{1}}$ and $\mathbf{r}_{2} \mathbf{e}^{-\mathrm{j} \phi_{2}}$ are the reflection coefficients of the mirrors, $\mathbf{t}_{1}$ is the transmission coefficient of the front mirror, $\beta$ is the propagation constant for the traveling EM wave in air, and $\mathbf{L}$ is the separation width of the cavity. The backward traveling wave $\mathrm{E}_{\mathrm{b}}$ is related to $\mathrm{E}_{\mathrm{f}}$ as:

$$
E_{b}=r_{2} e^{-\alpha d} e^{-j\left(\beta L+\phi_{2}\right)} E_{f}
$$

Using Eqs. (1) and (2), we can calculate the power enhancement factor $\eta$, which is defined as the ratio of the absorbed power inside the absorption layer, to the power of the incident EM wave,

$$
\eta=\frac{\left(1+R_{2} e^{-\alpha \mathrm{d}}\right)\left(1-R_{1}\right)}{1-2 \sqrt{R_{1} R_{2}} e^{-\alpha \mathrm{d}} \cos \left(2 \beta L+\phi_{1}+\phi_{2}\right)+R_{1} R_{2} e^{-2 \alpha \mathrm{d}}},
$$

where $\mathbf{R}_{1}=\mathbf{r}_{1}{ }^{2}$ and $\mathbf{R}_{2}=\mathbf{r}_{2}{ }^{2}$, are the reflectivities of the mirrors of the cavity. The above result is normalized with respect to the incident field absorbed by the detector in the absence of the crystal. The aforementioned planar defect structure have symmetric mirrors where $\mathbf{R}=\mathbf{R}_{\mathbf{1}}=\mathbf{R}_{\mathbf{2}}$. We used the measured transmission characteristics to obtain the reflectivities of our photonic mirrors. As the rods are made of high quality alumina with a very low absorption coefficient, the absorption in the crystal can be neglected. ${ }^{20}$ At the defect frequency, the transmission of an 8-layer crystal was $30 \mathrm{~dB}$ below the incident EM wave. The reflectivity of the photonic mirrors was then obtained as $\mathbf{R}=\mathbf{1 - T}=\mathbf{0 . 9 9 9}$. The ideal case which maximizes $\eta$ corresponds to $\boldsymbol{\alpha d}=\mathbf{0}$, which gives a maximum enhancement factor of 2000 . We then varied $\boldsymbol{\alpha d}$ to obtain enhancement factors closer to our experimental measurements. For $\boldsymbol{\alpha d}=\mathbf{0 . 0 0 0 1}$, Eq.(3) yields an enhancement factor of 1600 (which corresponds to the value obtained from the network analyzer), while $\boldsymbol{\alpha d} \mathbf{0 0 . 0 0 1 1}$ results in an enhancement factor of $\mathbf{4 5 0}$ (microwave detector). The increased absorption factor for the detector measurement can be explained by the relatively large volume size of the microwave detector compared to monopole antenna alone. Figure 4 compares the measured (solid line) and simulated (dotted line) enhancements obtained for the RCE microwave detector within the planar defect structure. The theoretical Q-factor (1500) is comparable with the experimental Q-factor (1100). 


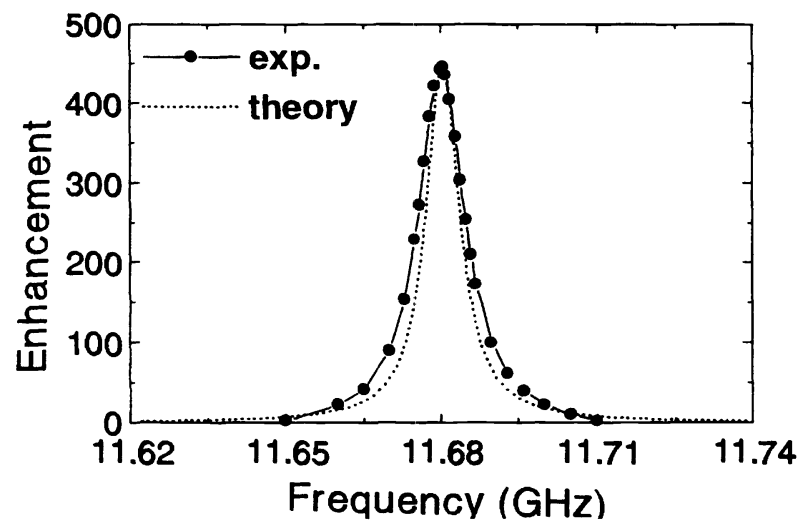

Figure 4. Comparison of the experimental (solid line) and theoretical (dotted line) enhancement factors obtained for the $\mathrm{RCE}$ detector in the planar defect structure.

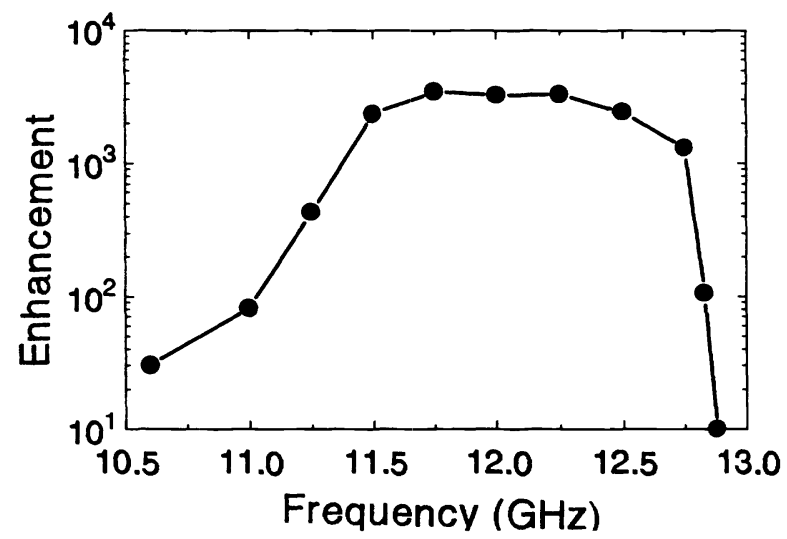

Figure 5. The power enhancement (given in logarithmic scale) can be obtained at different resonant frequencies by changing the cavity width. This corresponds to a tuning bandwidth ranging from $10.5 \mathrm{GHz}$ to $12.8 \mathrm{GHz}$.

\section{TUNABLE DETECTORS}

The Fabry-Perot model suggests that $\eta$ is maximized for the matching case $\mathbf{R}_{\mathbf{1}}=\mathbf{R}_{\mathbf{2}} \mathbf{e}^{-2 \alpha d}{ }^{22}$ To increase the enhancement, we increased $\mathbf{R}_{\mathbf{2}}$ by adding one more unit cell (4 layers) to the mirror at the back. This result in an asymmetric planar cavity with a 2 unit cell thick front mirror, and a 3 unit cell thick back mirror. By varying the width of the planar cavity, we measured the enhancement factors at different resonant frequencies. As shown in figure 5, the tuning bandwidth of the RCE detector extends from $10.5 \mathrm{GHz}$ to $12.8 \mathrm{GHz}$. This tuning bandwidth of the RCE detector is in good agreement with the full photonic band gap (10.6-12.7 GHz) of the crystal. ${ }^{15}$ As expected, the measured enhancement factors are relatively higher when compared with the symmetrical defect case. The maximum enhancement was measured to be 3450 at a defect frequency of $11.75 \mathrm{GHz}$. The theory predicted enhancement factors around 5500 , which is higher than the measured values. The discrepancy can be explained by the finite size of the photonic crystal, which limits the power enhancement of the field within the cavity. 


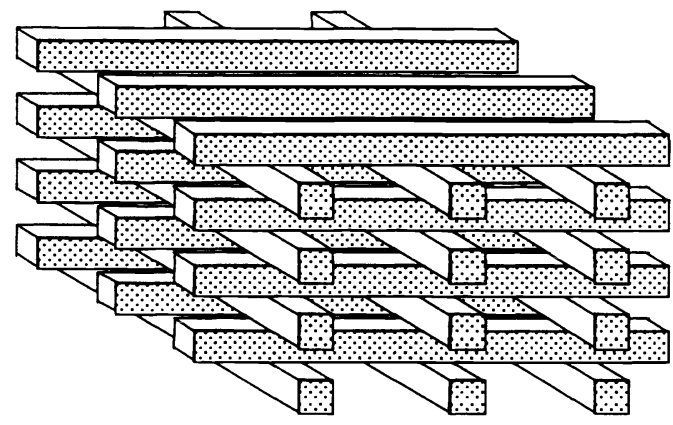

Figure 6. Schematics of simple tetragonal based photonic crystals.

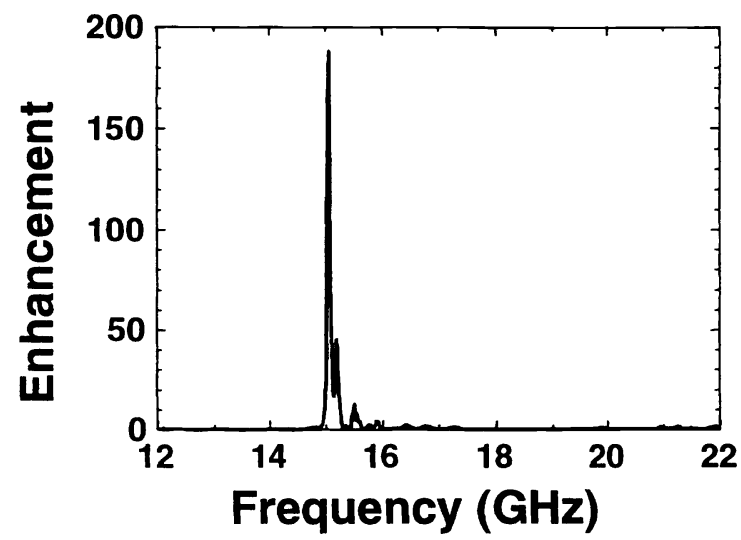

Figure 7. Enhancement characteristics of a planar defect structure within 8 layer st based metallic photonic crystal.

\section{METALLIC STRUCTURES}

The metals are perfect reflectors at microwave frequencies, and EM waves can not penetrate into these materials. This property results in the metallicity gap which is expected to extend down to $0 \mathrm{GHz}^{21-23}$ Figure 6 shows the structure we used for metallic photonic crystals. This the other structure is similar to the fct structure shown in Figure 1, but with a repeating sequence of 2 layers, which is the analog of a simple tetragonal (st) lattice. The metallic rods used in these crystals were 0.8 $\mathrm{mm}$ wide, $2.5 \mathrm{~mm}$ thick, and $120 \mathrm{~mm}$ long, and were placed with center to center separation of $7.6 \mathrm{~mm}$. The rods were obtained by machining $150 \times 150 \times 5 \mathrm{~mm}$ aluminum blocks. These blocks were then stacked together to form either fct or st structures depicted in Fig. 6. Figure 7 shows the enhancement characteristics of a planar type of defect structure, with a 4 layer thick front mirror and a 6 layer thick back mirror. At a $5 \mathrm{~mm}$ separation width of the cavity, the defect frequency was observed to be at $15.08 \mathrm{GHz}$. We observed a power enhancement factor of 190 at the defect frequency, with a Q factor of 335. When compared with enhancement factors obtained in cavity structures built around dielectric photonic crystals, this value is rather small. This may be the result of small but finite absorption coefficients of metals, which exists even at microwave frequencies. This absorption becomes significant when we consider the high number of times the field circulates inside the cavity and experiences a loss in each cycle. Still these investigations suggest the possibility of using embedded detector inside a metallic photonic crystal, as a frequency selective RCE detector with an increased sensitivity and efficiency. As an example, detectors based on photonic crystals will be more efficient than a typical frequency selective LC resonant circuit detector as the EM field does not recycle in such detectors. The RCE detectors based on photonic crystals are also superior to other type of resonant cavity antennas such as patch antennas. The ultra-high EM wave rejection properties photonic crystals result in better and more controlled confinement of the resonant EM field in all directions. This means photonic crystal based RCE detectors will have higher quality factors and better frequency selective properties when compared to conventional RCE detectors. 


\section{ACKNOWLEDGEMENTS}

This work is supported by the Turkish Scientific and Technical Research Council of TURKEY (TÜBITAK) under contract No. 197-E044, NATO Grant No. SfP971970, National Science Foundation Grant No. INT-9512812, and NATOCollaborative Research Grant No. 950079.

\section{REFERENCES}

1. K. M. Ho, C. T. Chan, and C. M. Soukoulis, Phys. Rev. Lett. 65, pp. 3152-3155, 1990.

2. E. Yablonovitch, T. J. Gmitter, and K. M. Leung, Phys. Rev. Lett. 67, pp. 2295-2298, 1991.

3. S. John, Phys. Rev. Lett. 58, 2486 (1987).

4. Photonic Band Gap Materials, C.M. Soukoulis, Plenum, New York, 1996.

5. J. D. Joannopoulos, P. R. Villeneuve, and S. Fan, Nature 386, pp. 143-144, 1997.

6. J. D. Joannopoulos, R. D. Meade, J. N. Winn, Photonic Crystals, Princeton University Press, 1995.

7. V. Kuzmiak, and A. A. Maradudin, Phys. Rev. B 55, pp. 7427-7430, 1997.

8. M. M. Sigalas, C. T. Chan, K. M. Ho, and C. M. Soukoulis, Phys. Rev. B 52, pp. 11744-11750, 1995.

9. D. F. Sievenpiper, E. Yablonovitch, J. N. Winn, S. Fan, P. R. Villeneuve, J. D. Joannapoulos, Phys. Rev. Lett. 80, pp. 2829-2832, 1998.

10. M. J. Bloemer, and M. Scalora, Appl. Phys. Lett. 72, pp. 1676-1678, 1998.

11. C. C. Cheng, Rong-Chung Tyan, A. Scherer, Y. Fainman, G. Witzgall, E. Yablonovitch, J. Vac. Sci. Technol. B 15, pp. 2764-2767, 1997.

12. J. C. Knight, T. A. Birsk, P. St. J. Russel, and D. M. Atkin, Optics Letters 21, pp. 1547-1549, 1996.

13. K. M. Ho, C.T. Chan, C. M. Soukoulis, R. Biswas, and M. Sigalas, Solid State Commun. 89, pp. 413-416, 1994.

14. E. Ozbay, J. Opt. Soc. Am. B 13, pp.1945-1954, 1996.

15. M. C. Wanke, O. Lehmann, K. Muller, Q. Wen, M. Stuke, Science 275, 1284 (1997).

16. S.Y. Lin, J.G. Fleming, D.L. Hetherington, B.K. Smith, R. Biswas, K.M. Ho, M.M. Sigalas, W. Zubrzycki, S.R. Kurtz, J. Bur, Nature 394, pp. 251-252, 1998.

17. E. Ozbay, G. Tuttle, M. Sigalas, C.M. Soukoulis, and K.M. Ho, Phys. Rev. B 51, pp. 13961-13965 (1995).

18. A.E. Siegman, Lasers, University Science Books, Mill Valley, 1986.

19. M. Selim Unlu, and S. Strite, J. Appl. Phys. 78 pp. 1-31, 1995.

20. E. Ozbay, B. Temelkuran, Appl. Phys. Lett. 69, pp. 743-745, 1996.

21. D. F. Sievenpiper, M.E. Sickmiller, E. Yablonovitch, Phys. Rev. Lett. 76, pp. 2480-2483, 1996.

22. E. Ozbay, B. Temelkuran, M. Sigalas, G. Tuttle, C. M. Soukoulis, and K. M. Ho, Appl. Phys. Lett. 69, pp. 3797-3799, 1996.

23. J. S. McCalmont, M. Sigalas, G. Tuttle, K. M. Ho, and C. M. Soukoulis Appl. Phys. Lett. 68, pp. $2759-2761,1996$. 